\title{
Caries dental en niños con necesidades especiales de un colegio de bajos recursos en el Perú
}

\author{
Dental caries in children with special needs from a low resource school in Peru
}

\author{
Diana del Carmen Muñoz Cárdenas ${ }^{1 a, 2, b}$, Lillie Elizabeth Abanto Silva ${ }^{3, c, d, e}$, Roberto Antonio León-Manco ${ }^{4, c, f, e}$, \\ Carol Mónica Zavaleta Boza ${ }^{4, c e, g}$
}

\section{RESUMEN}

Objetivos: Determinar la prevalencia de caries dental en los niños del centro de educación básica especial Helen Keller situado en el Callao, Perú durante el 2015. Material y Métodos: Estudio observacional, transversal sobre los datos de 30 niños del centro de educación básica especial Helen Keller. Información recogida por alumnos de la Facultad de Estomatología de la Universidad Peruana Cayetano Heredia (UPCH), en el centro educativo durante el año 2015. Se analizaron las características de los niños (edad, sexo, y diagnóstico sistémico). Para caries dental se utilizó el índice CPOD/ceod y el índice CPOS/ceos. Resultados: El 90\% (n=27) de los escolares presentó caries dental. El retardo mental fue la condición más prevalente (43,3\%, n=13). Se encontró un índice de CPOD de 2,9 (DE 3,8) y en dientes deciduos (ceod) de 6,0 (DE 4,0). Conclusiones: Existe una alta prevalencia de caries dental en los niños del centro de educación básica especial Helen Keller Callao, Perú en el año 2015.

PALABRAS CLAVE: Niño, discapacidad intelectual, caries dental, atención dental para personas con discapacidades.

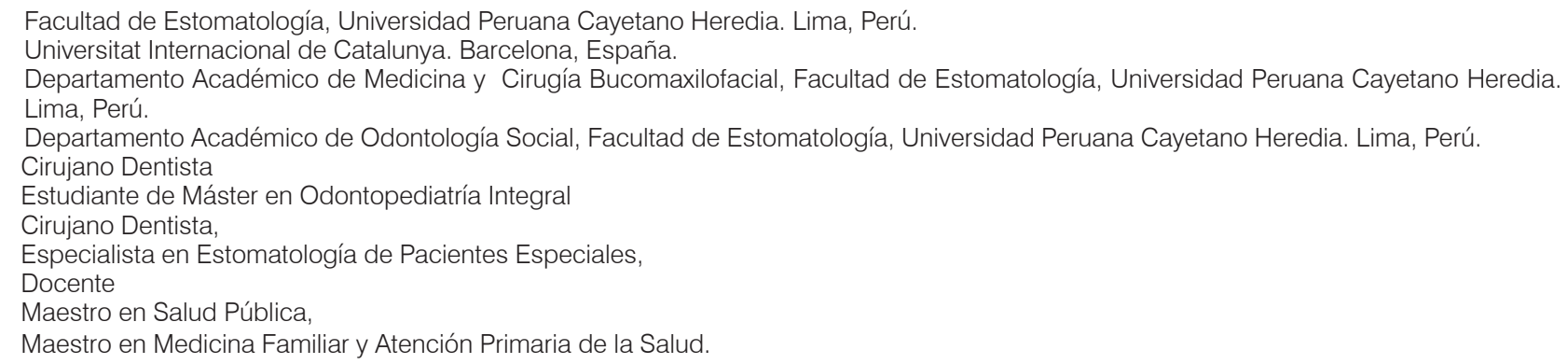




\section{SUMMARY}

Objectives: Determine dental caries prevalence of children from a basic educational center for special needs, Helen Keller school situated at Callao, Perú during 2015. Material and Methods: Cross-sectional study on a sample of 30 children from the basic educational center for special needs, Helen Keller school. The data was collected from Universidad Peruana Cayetano Heredia (UPCH) during 2015 at mentioned school. Children characteristics were analyzed (age, sex, systemic diagnosis) in frequencies, and dental caries prevalence. We analyzed dental caries per each tooth (DMFT/dmft index) and each surface (DFMS/dmfs index). Results: 90\% $(n=27)$ of scholars had dental caries. Mental retardation, was the most common condition $(43.3 \%, n=13)$. We found a DMFT index of 2.0 (SD 3.8) and in deciduous teeth (dmft index) of 6.0 (SD 4.0). Conclusions: There is a considerable proportion of children that have dental caries in Basic educational center for special needs Helen Keller, Callao, Perú during 2015.

KEY WORDS: Child, intellectual disability, dental caries, dental care for disabled.

\section{INTRODUCCIÓN}

Las enfermedades bucales más prevalentes, no son diferentes en poblaciones con características diversas. Existe mayor prevalencia de enfermedades bucales en poblaciones con necesidades especiales, porque cuentan con un menor acceso al servicio de salud y mayor probabilidad de enfermarse. Una de estas poblaciones, son las personas con necesidades especiales. Una de las razones más importantes por lo que esta población presenta problemas dentales es debido a una deficiencia en la higiene oral. Además, se encuentran mayores problemas periodontales y prevalencia de caries dental. Consecuentemente, el cuidado en estas personas debe ser mayor al común, ya sea por factores como el uso de fármacos, como también características propias de la patología que presenten (1). Además, se debe de tener en cuenta que las personas con necesidades especiales no dependen de ellos mismos, sino sus padres o cuidadores y son ellos los responsables de llevar a cabo toda actividad preventiva (2). Sin embargo, las personas a cargo del cuidado de estos pacientes son conscientes de que una persona con una necesidad especial posee características orales asociadas a su enfermedad (i.e. sangrado gingival, caries dental, y halitosis), refieren no recibir información acerca del cuidado de la salud oral (3).

Los tratamientos en la salud bucal son una necesidad desatendida en relación al tratamiento médico de estas personas (4). Dos razones pueden explicar esta falencia. La primera es una carencia de coordinación en relación a los servicios de salud y la segunda la falta de conocimiento de los profesionales de salud con respecto a esta área $(4,5)$.

Existe poca información reportada en la literatura en nuestro país sobre el estado de salud oral de los pacientes con necesidades especiales.

El propósito de esta investigación fue determinar la prevalencia de caries dental de los niños del centro de educación básica especial Helen Keller situado en el Callao, Perú durante el 2015.

\section{MATERIAL Y METODOS}

El diseño del estudio fue descriptivo, retrospectivo y observacional. Se utilizaron datos de una fuente secundaria que recolectó información sobre escolares (de 5 hasta 18 años de edad) del centro de educación básica especial Helen Keller. La información fue recopilada el 2015 por el Departamento Académico de Odontología Social y el servicio de Estomatología de Pacientes Especiales-Departamento Académico de Medicina y Cirugía Bucomaxilofacial, de la Universidad Peruana Cayetano Heredia (UPCH). El cual recogió información acerca de la edad y sexo de los niños según lo estipulado por el centro de educación básica especial Helen Keller. Ademas, la información acerca del diagnóstico sistémico lo recopilaron de los registros del centro educativo. Por otro lado, las examinaciones orales se realizaron siguiendo el protocolo establecido por la Organización Mundial de la Salud (OMS), utilizando como indicador de caries dental el CPOD para dientes permanentes y el ceod para dientes deciduos, con consentimiento de los padres y/o tutores legales, fueron examinados todos los niños del colegio, siendo un total de 37. De los cuales, 
7 (19\%) se excluyeron de este estudio por no tener información completa de sus historiales médicos, por lo que la muestra total del estudio fue de 30 niños (81\%).

La información de los escolares fue brindada con permiso del Departamento Académico de Odontología Social de la UPCH. Este estudio fue aprobado por el Comité de Ética de la UPCH.

La edad de los niños fue registrada de forma cuantitativa y el sexo de forma cualitativa. Los diagnósticos sistémicos (síndrome) fueron categorizados de forma dicotómica, si el niño presentaba o no deficiencia visual cerebral, deficiencia auditiva, autismo, síndrome de Down, parálisis cerebral y retardo mental. Por otro lado, la caries dental se midió como prevalencia de forma dicotómica, si el niño presentaba al menos una lesión de caries en boca. Y como experiencia de caries dental por medio de los índices ceod y CPOD, se registró de forma cuantitativa continua el promedio de dien- tes cariados, perdidos u obturados y sus superficies.

El análisis del estudio fue descriptivo. Se analizó las características de los niños mediante frecuencias relativas y absolutas de las variables cualitativas, así como, el promedio y desviación estándar de las variables cuantitativas.

\section{RESULTADOS}

De los 30 niños del centro de educación básica especial Helen Keller, la condición de retardo mental (43,3\%, n=13), fue el diagnóstico sistémico más prevalente, por el contrario el menos predominante fue el autismo $(3,3 \%, \mathrm{n}=1)$ y deficiencia auditiva $(3,3 \%$, $\mathrm{n}=1$ ). La edad promedio fue de 9.1 años, con una desviación estándar (DE) de 4,3. Además, el 76,7\% $(n=23)$ fueron de sexo masculino y el 23,3\% $(n=7)$ del sexo femenino (tabla 1 ).

Tabla 1. Descripción de la población de estudio de los niños del centro de educación básica especial Helen Keller, distrito de Ventanilla, Callao-Perú, en el año 2015.

\begin{tabular}{|c|c|c|}
\hline CARACTERÍSTICAS & $\mathbf{n}$ & $\%$ \\
\hline \multicolumn{3}{|l|}{ Diagnóstico sistémico } \\
\hline Deficiencia visual cerebral & 9 & 30,0 \\
\hline Deficiencia auditiva & 1 & 3,3 \\
\hline Autismo & 1 & 3,3 \\
\hline Síndrome de Down & 2 & 6,7 \\
\hline Parálisis cerebral & 4 & 13,3 \\
\hline Retardo mental & 13 & 43,3 \\
\hline Edad* (en años) & 9,1 & 4,3 \\
\hline \multicolumn{3}{|l|}{ Sexo } \\
\hline Femenino & 7 & 23,3 \\
\hline Masculino & 23 & 76,7 \\
\hline
\end{tabular}

N: Población.

n: Frecuencia absoluta.

$\%$ : Frecuencia relativa.

*: Se ha calculado el promedio y desviación estándar por ser variable cuantitativa. 
El 90\% ( $n=27)$ de los niños presentó al menos una lesión de caries dental en dientes deciduos o permanentes. En los permanentes, el CPOD fue de 2,9 (DE 3,8) en promedio. Ésta, fue compuesta por un promedio de 2,9 (DE 3,8) dientes cariados, y 0,0 (DE 0,2) de dientes perdidos. Ningún niño presentó obturaciones. En los dientes deciduos, el índice ceod tuvo un promedio de 6,0 (DE 4,0). Correspondiendo a una proporción de 5,7 (DE 4,0) dientes cariados del total, un promedio de 0,2 (DE 0,9) dientes cariados y de dientes obturados de 0,1 (DE 0,4). El ceos (superficies de los dientes) tuvo un valor de 11,0 (9,8\%), de las cuales 9,9 (8,1\%) correspondieron a superficies con caries, 0,9 (4,0\%) a extraídas y $0,2(0,7 \%)$ a obturadas. En relación a la caries dental según el sexo. Los niños tuvieron una mayor prevalencia de caries dental $(70 \%, \mathrm{n}=21)$. Por otro lado, la caries dental en las niñas fue del 20,0\% ( $n=6)$. El índice de CPOD fue similar para los niños (2,9, DE 4,3) que para las niñas (3,0, DE 2,2). Sin embargo, en

Tabla 2. Prevalencia de caries dental según sexo de los niños del centro de educación básica especial Helen Keller, distrito de Ventanilla, Callao-Perú, en el año 2015.

\begin{tabular}{|c|c|c|c|c|c|c|}
\hline & \multicolumn{4}{|c|}{ SEXO } & & \\
\hline & \multicolumn{2}{|c|}{ Femenino } & \multicolumn{2}{|c|}{ Masculimo } & \multicolumn{2}{|c|}{ Total } \\
\hline & $\mathbf{n}$ & $\%$ & $\mathbf{n}$ & $\%$ & $\mathbf{n}$ & $\%$ \\
\hline \multicolumn{7}{|l|}{ Caries dental } \\
\hline Con caries dental & 6 & 20,0 & 21 & 70,0 & 27 & 90,0 \\
\hline Sin caries dental & 1 & 3,3 & 2 & 6,7 & 3 & 10,0 \\
\hline \multicolumn{7}{|c|}{ Caries dental dientes deciduos } \\
\hline dc & 3,5 & 3,3 & 6,2 & 4,0 & 5,7 & 4,0 \\
\hline de & 0,0 & 0,0 & 0,3 & 1,0 & 0,2 & 0,9 \\
\hline do & 0,0 & 0,0 & 0,1 & 0,5 & 0,1 & 0,4 \\
\hline ceod & 3,5 & 3,3 & 6,6 & 4,0 & 6,0 & 4,0 \\
\hline sc & 7,3 & 7,9 & 10,6 & 8,3 & 9,9 & 8,1 \\
\hline se & 0,0 & 0,0 & 1,1 & 4,5 & 0,9 & 4,0 \\
\hline so & 0,0 & 0,0 & 0,2 & 0,8 & 0,2 & 0,7 \\
\hline ceos & 7,3 & 7,9 & 11,9 & 10,3 & 11,0 & 9,8 \\
\hline \multicolumn{7}{|c|}{ Caries dental dientes permanentes } \\
\hline DC & 3,0 & 2,2 & 2,9 & 4.2 & 2,9 & 3,8 \\
\hline $\mathrm{DP}$ & 0,0 & 0,0 & 0,0 & 0,2 & 0,0 & 0,2 \\
\hline DO & 0,0 & 0,0 & 0,0 & 0,0 & 0,0 & 0,0 \\
\hline CPOD & 3,0 & 2,2 & 2,9 & 4,3 & 2,9 & 3,8 \\
\hline $\mathrm{SC}$ & 4,4 & 3,9 & 4,5 & 7,9 & 4,5 & 7,0 \\
\hline SP & 0,0 & 0,0 & 0,2 & 1,1 & 0,2 & 0,9 \\
\hline $\mathrm{SO}$ & 0,0 & 0,0 & 0,0 & 0,0 & 0,0 & 0,0 \\
\hline CPOS & 4,4 & 3,9 & 4,7 & 8,1 & 4,6 & 7,2 \\
\hline
\end{tabular}

N: Población.

n: Frecuencia absoluta.

$\%$ : Frecuencia relativa.

*: Se ha calculado el promedio y desviación estándar por ser variable cuantitativa. 
los dientes deciduos los niños tuvieron un mayor índice de ceod $(6,6, \mathrm{DE} 4,0)$, en comparación con el sexo femenino (3,5, DE 3,3) (tabla 2) y gráfico 1.

Además, se puede observar un pico de aumento de caries dental en dientes deciduos para cuando el niño alcanza los 7 años de edad (ceod=7,3), y un punto más bajo cuando cumplen los 10, 11 años (ceod=3,0) (gráfico 2). Por otro lado, los niños de 12 y 18 años tuvieron el pico más alto de caries dental en dientes permanentes (CPOD 10,0). El pico menor fue alcanzado para cuando los niños tuvieron 6 años de edad (CPOD 0.2) (gráfico 3).

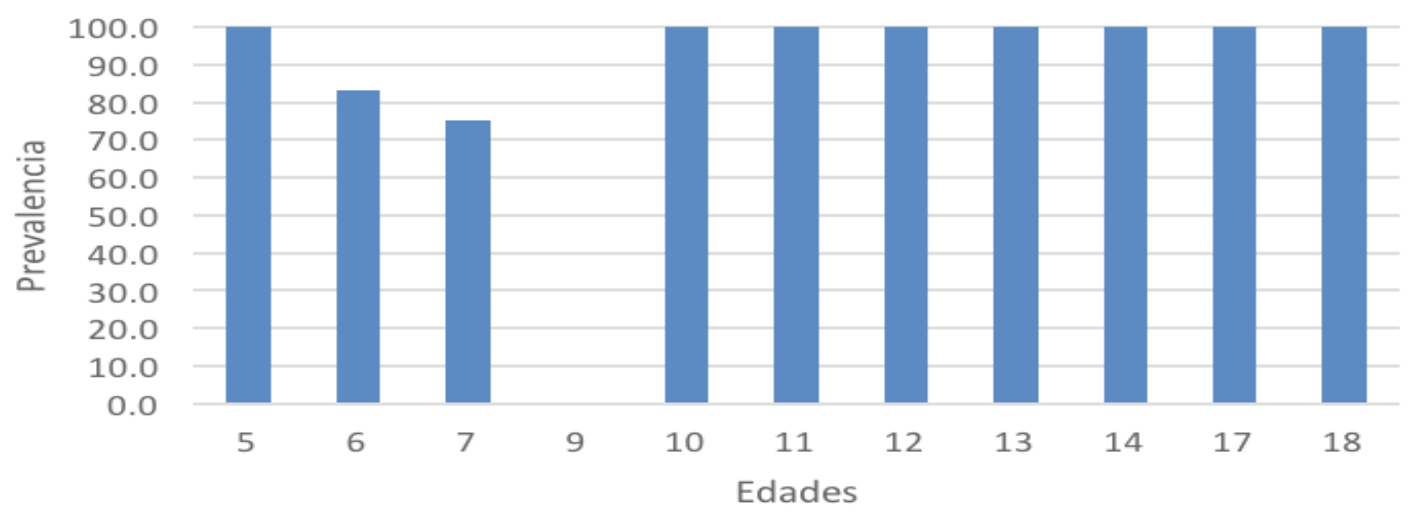

Gráfico 1. Aumento de la prevalencia de caries dental según la edad de los niños del centro de educación básica especial Helen Keller, distrito de Ventanilla, Callao-Perú, en el año 2015.

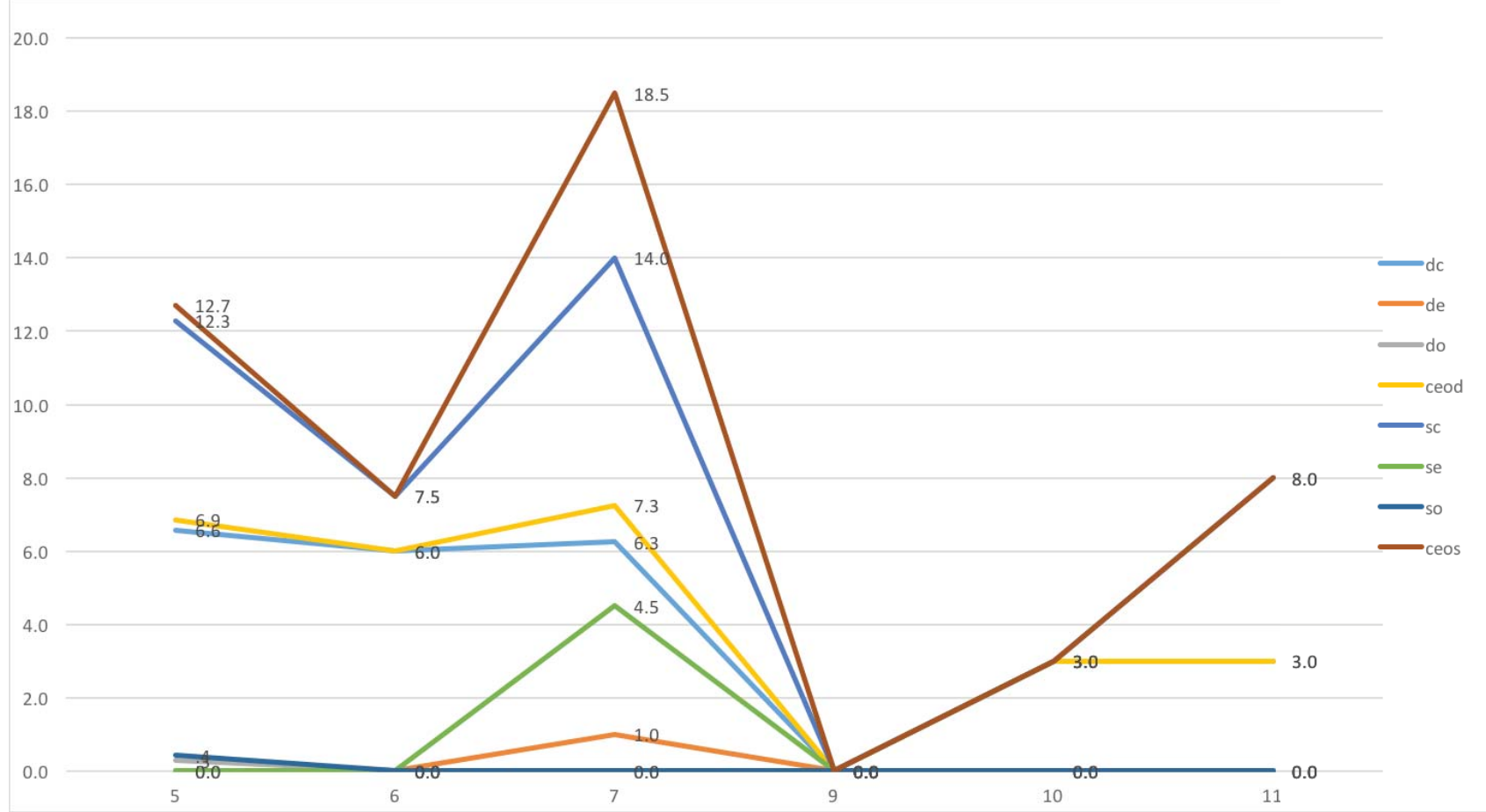

Gráfico 2. Caries dental, dientes perdidos y dientes obturados deciduos de los niños del centro de educación básica especial Helen Keller, Ventanilla-Callao en el año 2015 


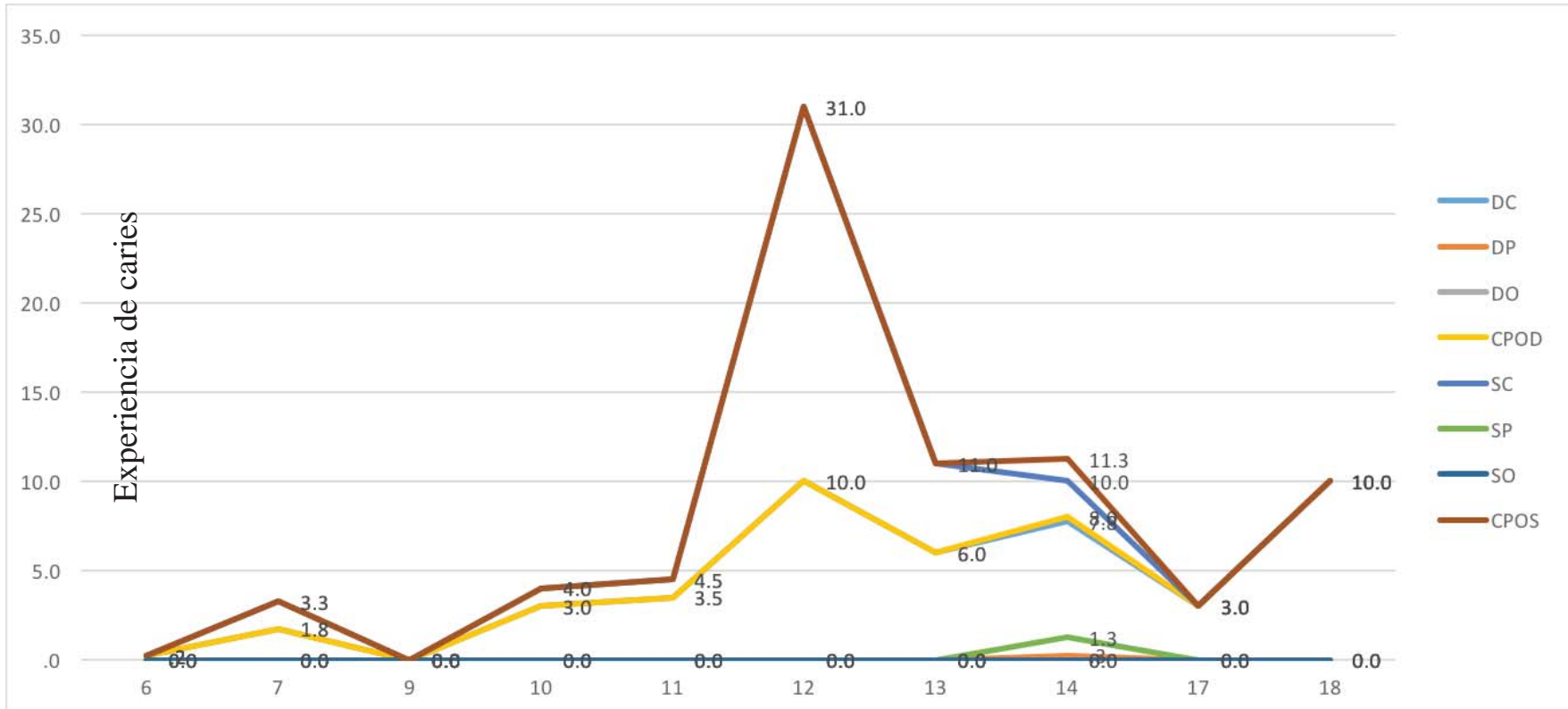

Gráfico 3. Caries dental, dientes perdidos, dientes obturados permanentes de los niños del centro de educación básica especial Helen Keller, Ventanilla-Callao en el año 201

\section{DISCUSIÓN}

En este estudio encontramos que la prevalencia de la caries dental fue de $(90 \%, \mathrm{n}=27)$,-mayormente en el sexo masculino $(70 \%, \mathrm{n}=21)$ en el centro de educación básica especial Helen Keller, localizado en el Callao, Perú durante el 2015.

En relación con el diagnóstico sistémico (síndrome), nuestros hallazgos son similares a otros estudios. En nuestro estudio se encontró que el retardo mental fue de mayor prevalencia (43,3\%). Cabellos en su estudio realizado en el centro Ann Sullivan Del Perú el año 2006, encontró que la prevalencia de retardo mental fue de $(43,3 \%)$ y el síndrome de Down fue $(14,4 \%)$ (6). Un estudio en Europa mostró que el retardo mental fue el síndrome más frecuente $(36,2 \%)$ y el menos prevalente el parálisis cerebral (4,3\%) (2). No obstante, los resultados no concuerdan con la investigación de Nelson et al., donde el autismo fue el más común (7), cabe resaltar, que el estudio mencionado no incluyó al retardo mental como diagnóstico.

Otro estudio que coincide con estos resultados es el de Vellappally et al., donde obtuvieron dentro de sus resultados que el $44,4 \%$ de su población total de estudio, eran niños con retardo mental (15), además, el Informe Mundial de la Discapacidad indica que las deficiencias que manifiesta una persona afectan su rendimiento en la escuela y en las tasas de matrícula. En donde los niños con una discapacidad física tienen una ventaja en este aspecto en comparación a los que padecen de una discapacidad intelectual o sensorial (8).

Por otro lado, las características de los niños en cuanto a la variable sexo son similares a los de otros estudio, como el de Paschal et al., que encontraron que los niños (sexo masculino) fue más prevalente $(60,1 \%)$ que en las niñas (sexo femenino) (39,9\%) (9). Nelson et al., encontraron que los niños de sexo masculino (64\%) tuvieron mayor prevalencia, donde los niños de sexo masculino formaron parte del $72,2 \%$ del estudio (10). Estos hallazgos, son similares a la presente investigación donde encontramos que los niños de sexo masculino tuvieron mayor prevalencia $(76,7 \%)$. Una de las razones que podrían explicar este fenómeno es que el sexo masculino es más frecuente en ciertos síndromes como el autismo o el síndrome de 
Down $(11,12)$. No obstante, el informe del centro latinoamericano del desarrollo (CELADE) indica que en las mujeres hay una mayor prevalencia de discapacidad, debido a una mayor longevidad (13). Por otro lado, la discriminación es importante de tomar en cuenta, siendo las mujeres las que sufren más de esto; por lo que en muchos casos son privadas de ir al colegio (8).

En cuanto a la edad, algunos estudios coincidieron con nuestros hallazgos, en niños menores de 14 años, la Carga Mundial de Morbilidad estima que en el mundo alrededor del 5\% (95 millones) padece de una discapacidad (8). En los adultos mayores, 65 años a más es la edad con mayor prevalencia de discapacidad (13). Los hallazgos encontrados en la literatura sobre la edad, se asemejan a los de la presente investigación donde la edad media fue de 9,1 años. En un estudio de Paschal et al., se halló una edad media de 10 años (9), sin embargo, los investigadores Oredugba et al., y Gaçe et al., hallaron una edad promedio de 12 años $(2,8)$. Otros estudios recopilaron la información sobre edad en forma de rangos; en este aspecto, Van Dyck et al., encontraron una mayor prevalencia de niños con necesidades especiales entre 12-17 años, siendo los menos prevalentes entre 0-5 años (14).

Con respecto a la caries dental un estudio realizado en el Perú, en 50 niños con retardo mental asistentes al Programa de Intervención Temprana del Hospital Regional Docente de Trujillo tuvo como resultado una alta prevalencia de caries (78\%), obteniendo un índice de ceod bajo (53,8\%).

En relación al nivel de conocimiento en los padres sobre higiene bucal y la higiene de los niños discapacitados, el resultado de una encuesta sobre conocimiento de higiene oral fue regular en el 68,9\% de los padres, seguido de un nivel de conocimiento malo (18,89\%), el $88,9 \%$ de los niños discapacitados presentaron un IHO, y no se reportó ningún niño con IHO bueno.

Es importante resaltar que no se han realizado investigaciones en el Perú sobre caries dental en las personas con necesidades especiales. En Nigeria, Oredugba et al., encontraron un índice de ceod de 0,7 y de CPOD de 0,4 en 54 personas de 3-26 años (10). En Estados Unidos, Gaçe et al., hallaron que la caries dental en los dientes permanentes fue alta $(85,3 \%, \mathrm{CPOD}=4,9)$ así también para los dientes decidua $(72 \%$, ceod=4,9), habiéndose realizado esta investigación en nueve colegios de niños especiales (2).

Nuestros hallazgos no coinciden con lo mencionado anteriormente, al obtener un CPOD de 2,9 y ceod de 6,0 en promedio respectivamente, estas diferencias pueden ser explicadas por un distinto tipo de población, el área de residencia en donde se recogieron los datos, y la importancia en la higiene oral enfatizada en los encargados de las personas con necesidades especiales.

Con esta investigación hemos podido demostrar la importancia de la salud oral en niños con necesidades especiales. Además, incentivar a profundizar en futuras investigaciones a nivel nacional en este campo de la salud oral en niños con necesidades especiales, y ser conscientes del rol fundamental que cumplen los padres o cuidadores de estos pacientes en cuanto a la salud oral.

El tipo de población que acude al centro de educación básica especial Helen Keller es de una zona rural, situada en la ciudad de Lima, es de escasos recursos económicos y con muy poco acceso a los servicios de salud.

\section{CONCLUSIONES}

Los niños del centro de educación básica especial Helen Keller, en el 2015, tuvieron una alta prevalencia de caries dental.

La caries dental de los niños del centro de educación básica especial Helen Keller aumentó con respecto a la edad.

El diagnóstico sistémico más prevalente de los niños con necesidades especiales del centro de educación básica especial Helen Keller, fue el de retardo mental.

\section{Correspondencia \\ Diana del Carmen Muñoz Cárdenas \\ Correo electrónico: diana.munoz.c@upch.pe}

\section{REFERENCIAS BIBLIOGRAFICAS}

1. Flores J, Carrillo D, Karzulovic L, et al. Niños y adolescentes con necesidades especiales de atención en salud: prevalencia hospitalaria y riesgos asociados. Rev Médica Chile. 2012;140:458-65.

2. Gaçe E, Kelmendi M, Fusha E. Oral health status of children with disability living in Albania. Mater Socio-Medica. 2014;26(6):392-4.

3. Martens L, Marks L, Goffin G, Gizani S, Vinckier F, Declerck D. Oral hygiene in 12-year-old disabled chil- 
dren in Flanders, Belgium, related to manual dexterity. Community Dent Oral Epidemiol. 2000;28(1):73-80.

4. Dávila L, Gil M, Daza D, Bullones X, Ugel G. Utilización de los servicios odontológicos por las personas con retraso mental con retraso mental en los municipios Irribarren y Palavecino. Bol Médco Postgrado. 2004;10(3).

5. Liu Z, Yu D, Luo W, et al. Impact of oral health behaviors on dental caries in children with intellectual disabilities in Guangzhou, China. Int J Environ Res Public Health. 2014;11(10):11015-27.

6. Cabellos D. Relación entre el nivel de conocimiento sobre higiene bucal de los padres y la higiene bucal del niño discapacitado en el centro Ann Sullivan del Perú. Tesis de Bachiller. Lima, Perú: Universidad Nacional Mayor de San Marcos; 2006.

7. Nelson L, Getzin A, Graham D, et al. Unmet dental needs and barriers to care for children with significant special health care needs. Pediatr Dent. 2011;33(1):29-36.

8. Organización Mundial de la Salud. World report on disability. Malta: OMS; 2011.

9. Paschal A, Wilroy J, Hawley S. Unmet needs for dental care in children with special health care needs. Prev Med Rep. 2016;3:62-7.

10. Oredugba F, Akindayomi Y. Oral health status and treatment needs of children and young adults attend- ing a day centre for individuals with special health care needs. BMC Oral Health. 2008;8:30.

11. Vishnu Rekha C, Arangannal P, Shahed H. Oral health status of children with autistic disorder in Chennai. Eur Arch Paediatr Dent Off J Eur Acad Paediatr Dent. 2012;13(3):126-31.

12. Otero J, Otero J.Odontología en pacientes con síndrome de down. Consultorio Médico; 2001. (Fecha de acceso 25 de abril de 2016) Disponible en: http://consultoriomedico.info/am.aspx?secc $=$ trafico\&id $=50$

13. Stang M. Las personas con discapacidad en América Latina: del reconocimiento jurídico a la desigualdad real. Santiago, Chile: Naciones Unidas; 2011 p. 1-85.

14. Van Dyck P, Kogan M, McPherson M, Weissman G, Newacheck P. Prevalence and Characteristics of Children With Special Health Care Needs. Arch Pediatr Adolesc Med. 2004;158(9):884.

15. Vellappally S, Gardens S, Al Kheraif A, et al. The prevalence of malocclusion and its association with dental caries among 12-18-year-old disabled adolescents. BMC Oral Health. 2014;14:123.

Recibido: 07-05-2018

Aceptado: 21-09-2018 